\title{
Steerable wavelet analysis of CMB structures alignment
}

\author{
Patricio Vielva ${ }^{1,2}$, Yves Wiaux ${ }^{3}$, \\ Enrique Martínez-González ${ }^{1}$ and Pierre Vandergheynst ${ }^{3}$ \\ ${ }^{1}$ Instituto de Física de Cantabria (CSIC - UC), Avda. Los Castros s/n, 39005, \\ Santander, Spain \\ 2 Astrophysics Group, Cavendish Laboratory, J J Thomson Avenue, Cambridge \\ CB3 OHE, UK \\ 3 Signal Processing Institute, Ecole Polytechnique Fédérale de Lausanne (EPFL), \\ CH-1015 Lausanne, Switzerland \\ E-mails:vielva@ifca.unican.es,yves.wiaux@epfl.ch,martinez@ifca.unican.es, \\ pierre.vandergheynst@epfl.ch
}

\begin{abstract}
This paper reviews the application of a novel methodology for analysing the isotropy of the universe by probing the alignment of local structures in the CMB. The strength of the proposed methodology relies on the steerable wavelet filtering of the CMB signal. One the one hand, the filter steerability renders the computation of the local orientation of the CMB features affordable in terms of computation time. On the other hand, the scale-space nature of the wavelet filtering allows to explore the alignment of the local structures at different scales, probing possible different phenomena. We present the WMAP first-year data analysis recently performed by the same authors (Wiaux et al.), where an extremely significant anisotropy was found. In particular, a preferred plane was detected, having a normal direction with a northern end position at $(\theta, \varphi)=\left(34^{\circ}, 331^{\circ}\right)$, close to the northern end of the CMB dipole axis. In addition, a most preferred direction was found in that plane, with a northern end direction at $(\theta, \varphi)=\left(71^{\circ}, 91^{\circ}\right)$, very close to the north ecliptic pole. This result synthesised for the first time previously reported anomalies identified in the direction of the dipole and the ecliptic poles axes. In a forthcoming paper (Vielva et al.), we have extended our analysis to the study of individual frequency maps finding first indications for discarding foregrounds as the origin of the anomaly. We have also tested that the preferred orientations are defined by structures homogeneously distributed in the sky, rather than from localised regions. We have also analysed the WMAP 3-year data, finding the same anomaly pattern, although at a slightly lower significance level.
\end{abstract}

Key words: cosmology, cosmic microwave background, data analysis

Preprint submitted to New Astronomy 26 September 2018 


\section{Introduction}

The release of the Wilkinson Microwave Anisotropy Probe (WMAP) firstyear data in 2003 (1) and the WMAP 3-year data in 2006 (3) have implied a huge activity in the Cosmic Microwave Background (CMB) community during the last years. Besides the impressive accuracy in the determination of the cosmological parameters given by the WMAP data analysis (2; 3) as well as by combining other cosmological data sets (e.g. (4)), a large effort was concentrated on the very challenging issue of probing the stationary Gaussian random nature of the CMB predicted by the standard inflationary Big-Bang model. This is a capital topic, since the determination of the cosmological parameters that define the concordance cosmological model relies on those assumptions.

Strong evidences (at confidence levels above 99\%) supporting the non-stationarity, or statistical anisotropy, of the CMB signal were established. Analyses based on N-point correlation functions $(5 ; 6)$, local curvature $(7)$, local power spectra $(8 ; 9 ; 10 ; 11)$, and bispectra $(12)$, suggest a north-south asymmetry maximised in a coordinate system with the north pole at $(\theta, \varphi)=\left(80^{\circ}, 57^{\circ}\right)$ in Galactic co-latitude $\theta$ and longitude $\varphi$, close to the north ecliptic pole lying at $(\theta, \varphi)=\left(60^{\circ}, 96^{\circ}\right)$. Analyses of multipole vectors, angular momentum dispersion, as well as azimuthal phases correlations find an anomalous alignment between the low $l$ multipoles of the $\mathrm{CMB}$, suggesting a preferred direction around $(\theta, \varphi)=\left(30^{\circ}, 260^{\circ}\right)$, near the ecliptic plane and close to the axis of the dipole lying at $(\theta, \varphi)=\left(42^{\circ}, 264^{\circ}\right)(13 ; 14 ; 15 ; 16 ; 17 ; 18 ; 19 ; 20)$. Galactic north-south asymmetries are also found in the analysis of the kurtosis and the area of the wavelet coefficients of the CMB data (21). These are mainly due to a very cold spot (the so-called cold spot) in the southern hemisphere $((21 ; 22 ; 23 ; 24 ; 25)$ and $(26)$ in this volume). First results with the angular pair separation method, which probes the statistical isotropy both in real and multipole space, also seem to support those results (27). In opposition to the previous results, bipolar power spectra analyses are consistent with no violation of the statistical isotropy of the universe $(28 ; 29 ; 30 ; 31)$. Finally, theoretical models for an anisotropic universe are being studied to account for the observed effects $(32 ; 33 ; 34 ; 35 ; 36 ; 37)$.

The method we introduced in (38) probes the statistical isotropy of the CMB in a novel way: the analysis of the alignment of structures of the CMB signal. By searching for the directions towards which the local CMB structures are mostly oriented, preferred directions in the sky may be defined. The method is based on the steerable wavelet decomposition of the CMB. On the one hand, the filter steerability renders affordable in terms of computation time the calculation of the local direction of the CMB structures at each position on the sky. On the other hand, the scale-space nature of the wavelet filtering allows the probe of 


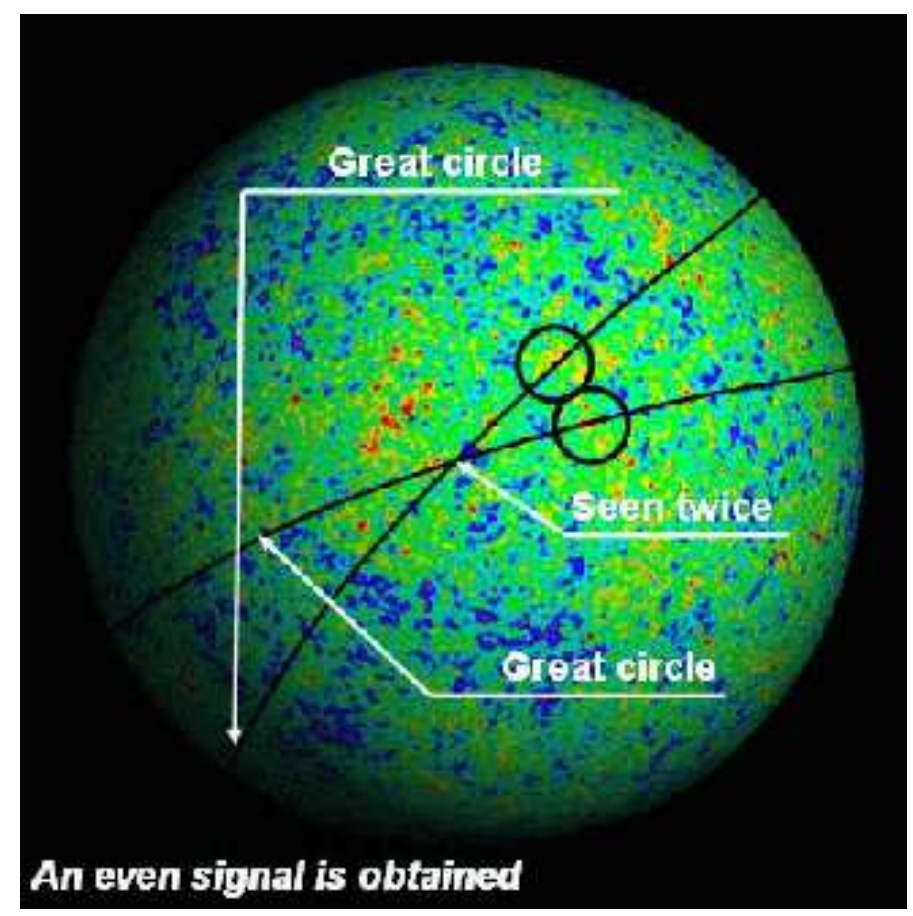

Fig. 1. Graphical illustration of the proposed methodology. The positions on the sphere towards which the local CMB structures are aligned are defined as those lying on the great circles that pass by the structures and are parallel to their orientations. The signal defined in this way is even (in Cartesian coordinates) by construction. This construction probes preferred directions in the sky towards which the CMB features are mostly oriented. As such, it represents a powerful analysis of the hypothesis of the universe isotropy. This figure is a modification of one from the Max Tegmark web site (http://space.mit.edu/home/tegmark/index.html).

the local CMB structures alignment as a function of the scale considered. Our analysis synthesised for the first time previously reported anomalies related with the dipole and the ecliptic poles axes. In this contribution we review our first work (38) as well as some new results from our forthcoming paper (39), relative of the detected anomaly. In Section 2 the details of the methodology are given. The application to WMAP data is detailed in Section 3. Finally, the Conclusions are in Section 4.

\section{Methodology}

Under the cosmological principle assumption, and in the framework of the basic inflationary models, the CMB can be interpreted as a stationary Gaussian random field on the sphere. The hypothesis of stationarity, or statistical isotropy, implies that no preferred orientation is expected. We propose to probe the statistical isotropy of the CMB by studying the orientation of its structures. The number of times a given direction in the universe is seen by the 


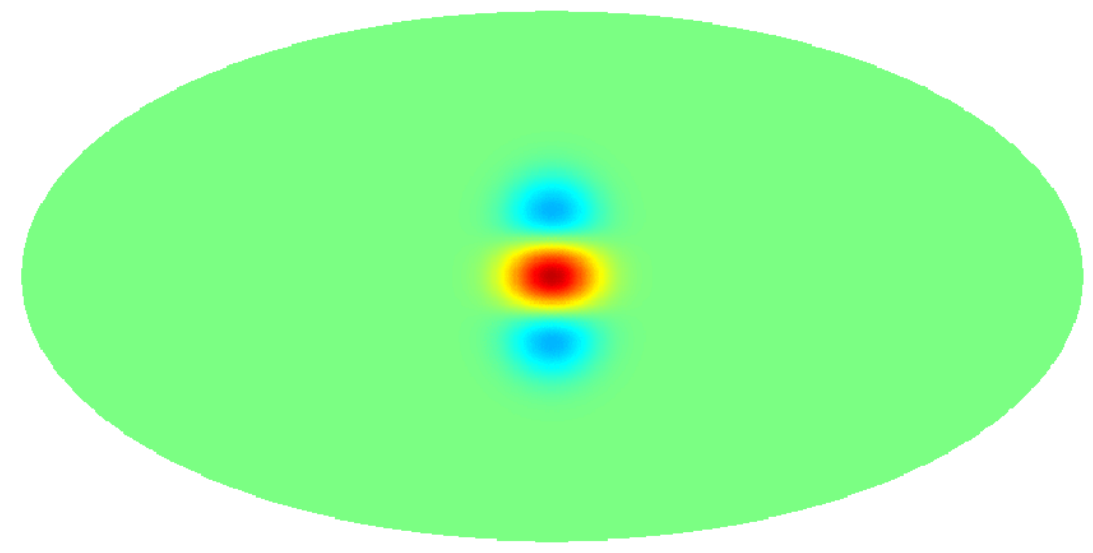

Fig. 2. Mollweide projection of the first member $\Psi_{\partial_{\hat{x}}^{2}, a}$ of the second Gaussian derivative (2GD) wavelet basis at position $\omega_{0}=\left(\theta_{0}, \varphi_{0}\right)=(\pi / 2,0)$ and scale $a=0.19$.

local $C M B$ features provides a unique way to determine whether there exists or not any preferred direction on the sky towards which the CMB structures are unexpectedly aligned.

An illustration of the proposed methodology is given in Figure 1. Let us focus on the two particular CMB structures in the middle of the two dark circles. All the positions on the sphere lying on the great circles that pass by the selected structures and are parallel to their orientations, are said to be seen by the corresponding structure. As it is indicated in the Figure, there is a particular position in the sky that is seen by both structures. Once this procedure is done for all the CMB structures, we end up with a signal on the sphere, allowing for a large number of statistical analyses (e.g. angular power spectrum, geometrical statistics, Minkowski functionals, ...). This signal is, by construction, even (in Cartesian coordinates), since it is obtained by analysing the direction of the features, without any notion about the specific sense.

The method is based on the steerable wavelet decomposition of the CMB signal. The reason for working in wavelet space is twofold. First, the wavelet filtering provides the natural framework for a multi-scale analysis of the local CMB structures. This opens the possibility to probe the statistical isotropy of the $\mathrm{CMB}$ at different angular resolutions, which is crucial since a possible source of anisotropy may be due to structures of a particular size. Second, steerable filters render the computation of the orientation of the features at each position on the sky affordable in terms of the computation time (40; 41). Steerable filters were firstly introduced on the plane by (42) and they have been recently extended to the sphere by (43). A non-axisymmetric filter is steerable if any rotation by $\chi \in[0,2 \pi)$ around itself may be expressed in terms of a finite linear combination of non-rotated basis filters. The reader 
is kindly referred to the previous references for a detailed description of the corresponding properties. The second Gaussian derivative (2GD) is a steerable wavelet filter, defined by the three following basis filters, at any scale $a>0$ :

$$
\begin{aligned}
\Psi_{\partial_{\hat{x}}^{2}, a}(\theta, \varphi) & =\frac{1}{a} \sqrt{\frac{4}{3 \pi}}\left(1+\tan ^{2} \frac{\theta}{2}\right) e^{-2 \tan ^{2}(\theta / 2) / a^{2}}\left[1-\frac{4}{a^{2}} \tan ^{2} \frac{\theta}{2} \cos ^{2} \varphi\right] \\
\Psi_{\partial_{\hat{y}}^{2}, a}(\theta, \varphi) & =\frac{1}{a} \sqrt{\frac{4}{3 \pi}}\left(1+\tan ^{2} \frac{\theta}{2}\right) e^{-2 \tan ^{2}(\theta / 2) / a^{2}}\left[1-\frac{4}{a^{2}} \tan ^{2} \frac{\theta}{2} \sin ^{2} \varphi\right] \\
\Psi_{\partial_{\hat{x}} \partial_{\hat{y}}, a}(\theta, \varphi) & =\frac{-2}{a} \sqrt{\frac{4}{3 \pi}}\left(1+\tan ^{2} \frac{\theta}{2}\right) e^{-2 \tan ^{2}(\theta / 2) / a^{2}} \sin (2 \varphi) \tan ^{2} \frac{\theta}{2},
\end{aligned}
$$

where the position $\omega$ at each point on the sphere is identified by its co-latitude $\theta \in[0, \pi]$ and longitude $\varphi \in[0,2 \pi)$. In Figure 2 we plot the first member of the basis, $\Psi_{\partial_{\hat{x}}^{2}, a}$, centred at position $\omega_{0}=\left(\theta_{0}, \varphi_{0}\right)=(\pi / 2,0)$, and scale $a=0.19$.

\section{Application to WMAP data}

We have applied the methodology outlined in Section 2 to the WMAP data. The results, obtained on the WMAP first-year data, where published in (38).

In a forthcoming paper (39), we extend the first analysis and present the results obtained for the three-year data. In this Section we summarise the most important results.

\subsection{Data and simulations}

Since we are interested in probing the CMB statistical isotropy, we need to deal with a CMB map as free of foreground emission and unknown systematics as possible. Many different CMB maps (all them obtained from the WMAP data) have been proposed and used during the last years. We considered the map defined by (44) in the WMAP Gaussianity paper. This map (the so-called template fits map) is generated as a noise-weighted combination of the eight temperature maps at the Q, V and W frequency channels, previously cleaned from foreground emission by performing a joint multi-template fitting (see (45) for details). After the combination, the so-called Kp0 mask is applied to account for diffuse Galactic emission close to the Galactic plane as well as for discarding bright point sources. Finally, the residual monopole and dipole are subtracted outside the mask. The resultant map is presented in Figure 3. The WMAP data are given in the HEALPix pixelization (46) at resolution 


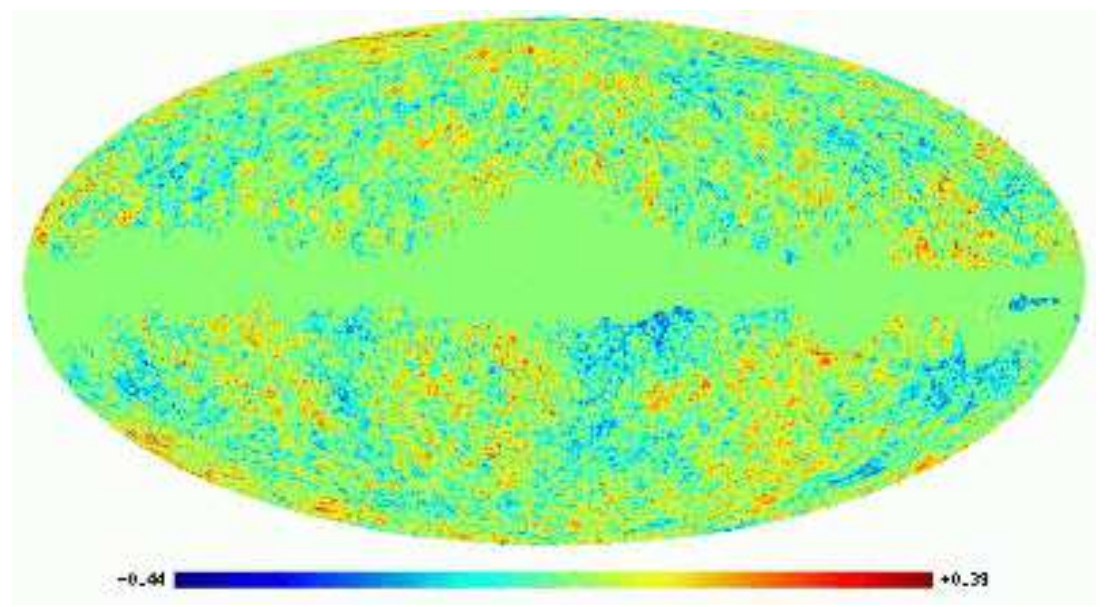

Fig. 3. The analysed CMB map obtained from the WMAP first-year data. This map is obtained by following the template fits method described in (45) and firstly used by (44) in the WMAP Gaussianity analysis.

parameters $N_{\text {side }}=512$ and $N_{\text {side }}=1024$. However, we have performed our analysis on maps downgraded at $N_{\text {side }}=32$, where the signal-to-noise ratio is extremely high. At that resolution, the sphere is defined by $N_{p i x}=12288$ pixels, with an angular resolution of $1.8^{\circ}$.

In order to confront the obtained results with the expected isotropic behaviour, we have performed 10000 simulations. For each simulation, a stationary Gaussian CMB realisation is generated from the theoretical angular power spectrum $C_{\ell}$ computed with CMBFAST (47), using the cosmological parameters given by the WMAP best-fit cosmological model. Each receiver is simulated by convolving the simulated map with the corresponding beam window function and by adding an anisotropic Gaussian noise realisation of the particular noise level. As for the data, the eight maps are linearly combined using weights defined by the noise amplitude per pixel. The Kp0 mask is applied, before removing the residual monopole and dipole.

\subsection{Results}

We have explored the CMB statistical isotropy at twelve different scales. For a given scale $a$ of the wavelet, the angular size of the 2GD wavelet is defined as twice the dispersion of the corresponding Gaussian. The twelve scales considered, correspond to angular sizes ranging from 5 to $50^{\circ}$. By convolving the CMB map with the wavelets, the pixels close to the mask are going to be very affected by the zero value of the mask. For that reason, at each scale, an extended exclusion mask $M_{a}$ is defined in order to avoid highly contaminated pixels close to the mask. For each pixel outside the mask in the sky and for each scale, the orientation for which the wavelet coefficient is maximum in absolute value is selected and the corresponding absolute value is retained. 


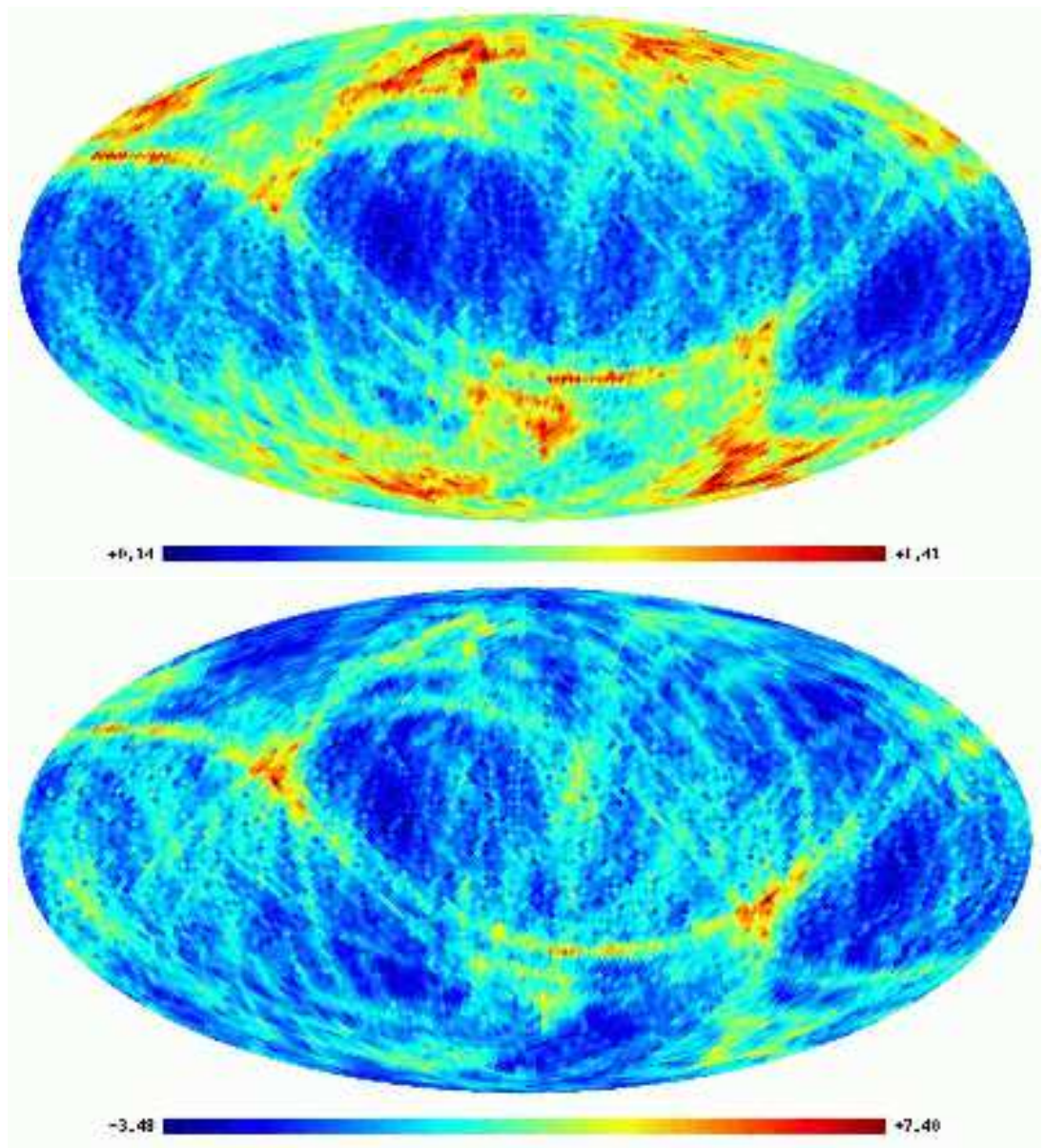

Fig. 4. Top panel: Map of total weights $D_{a_{3}}(\omega)$ resulting from the WMAP first-year data at scale $a_{3}$ corresponding to an angular size of $8.3^{\circ}$. Bottom panel, the same map, but expressed in $\sigma_{a_{3}}$ units (estimated from the 10000 simulations).

This procedure selects the local wavelet orientation that best matches the orientation of the local structure of the signal at each point.

As explained in (38), the total weight $D_{a}(\omega)$ is a weighted measure of the number of times a given pixel $\omega$ in the sky is seen by local features at a given scale $a$ in the original signal. It is defined as:

$$
D_{a}(\omega)=\frac{1}{A} \sum_{c=1}^{N_{\text {cros }}(\omega)}\left|W_{\Psi}^{F}\left(\omega_{0}^{(c)}, \chi_{0}\left(\omega_{0}^{(c)}\right), a\right)\right|
$$

where $W_{\Psi}^{F}\left(\omega_{0}, \chi_{0}\left(\omega_{0}\right), a\right)$ is the maximum value of the wavelet coefficient at the position $\omega_{0}$ (that is achieved at orientation $\chi_{0} \in[0,2 \pi)$ ) and the factor $A=L N_{p i x}^{-1} \sum_{\omega_{0} \notin M_{a}}\left|W_{\Psi}^{F}\left(\omega_{0}, \chi_{0}\left(\omega_{0}\right), a\right)\right|$ of normalisation defines a mean total weight in each direction equal to unity for isotropic CMB simulations without mask: $N_{p i x}^{-1} \sum_{\omega \in S} D_{a}(\omega)=1$. The quantity $L=4 N_{\text {side }}$ stands for the number of points on a great circle on a HEALPix grid. 


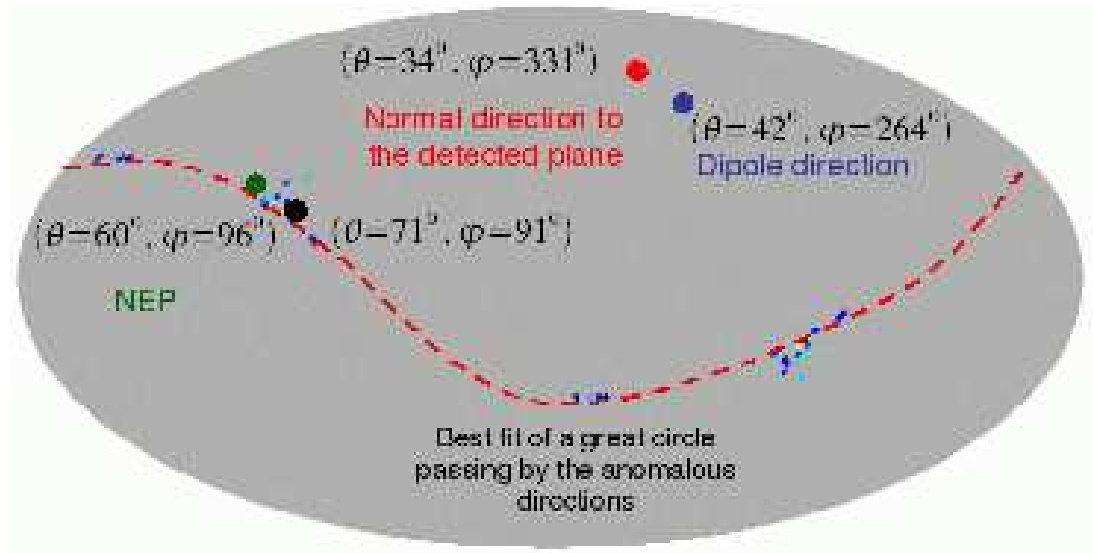

Fig. 5. The 20 anomalous directions (pairs of opposite points) with total weights higher than in any of the 10000 simulations are shown. They define a preferred plane with a perpendicular northern end position at $(\theta, \varphi)=\left(34^{\circ}, 331^{\circ}\right)$, very close to the $\mathrm{CMB}$ dipole axis at $(\theta, \varphi)=\left(42^{\circ}, 264^{\circ}\right)$ and to the so-called axis of evil. A preferred direction in the preferred plane is identified with a northern end position at $(\theta, \varphi)=\left(71^{\circ}, 91^{\circ}\right)$, which is extremely close to the north ecliptic pole at $(\theta, \varphi)=\left(60^{\circ}, 96^{\circ}\right)$

In the top panel of Figure 4 we present the signal $D_{a_{3}}(\omega)$ obtained from the analysis of the WMAP first-year data. The angular size corresponding to the scale $a_{3}$ is $8.3^{\circ}$. It is clear that the zone around the Galactic plane is less seen than the rest of the sky. It is an obvious result, since a pixel has more probability to be seen from nearby pixels than from distant ones. Hence the exclusion of pixels by the mask implies that, even in an isotropic universe, the pixels close to the mask are less seen than the others. Obviously, what is much more interesting is to represent the previous signal as normalised, at each point, by the standard deviation (sigma) estimated from the 10000 simulations, i.e. in $\sigma_{a}$ units. This normalisation notably cancel out all the spurious anisotropies, like the one due to the mask. This map is presented in the bottom panel. As it can be seen, there is a very particular pattern with many great circles crossing the equatorial poles. Obviously, the distribution of $D_{a_{3}}(\omega)$ is not Gaussian, and, therefore, the number of sigmas is not a direct measurement of the probability of each direction, although it is quite useful for a visual illustration. In any case, the probability associated with each direction in the sky (pairs of opposite points) can be easily computed from the simulations. In particular, there are 27 directions that present values of $D_{a_{3}}(\omega)$ anomalous at $99.99 \%$ (i.e., at maximum, only one simulation has a larger value). Even more, just one simulation has at least 27 directions above $99.99 \%$ at scale $a_{3}$.

Among the previous 27 directions, 20 present values of $D_{a_{3}}(\omega)$ larger than in any of the 10000 simulations. These directions (Figure 5) are also identified as being anomalous at more than $99.99 \%$ (a 100\% statement being prohibited by the finite size of our sample of simulations). These anomalous direc- 


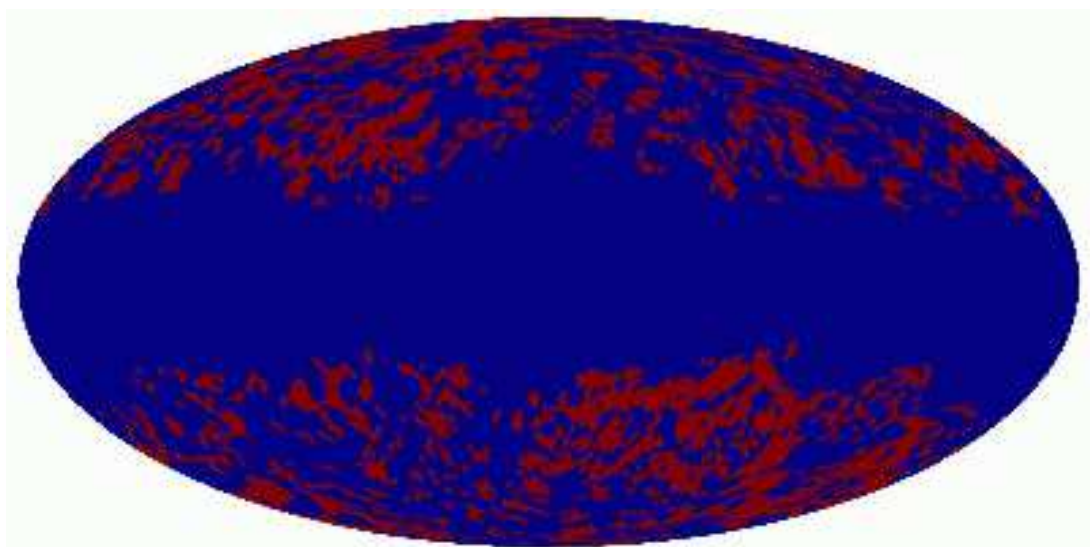

Fig. 6. The pixels that are aligned towards any of the 20 directions anomalous at more than $99.99 \%$ in the WMAP first-year data are shown (in dark red). They are homogeneously distributed in the sky, which seems to indicate that the source of the detected anomaly is not localised in a specific region of the sky.

tions are shown in Figure 5. They are mostly concentrated in two clusters of 4 and 16 directions. The mean direction ${ }^{1}$ of the latter has a northern end at $(\theta, \varphi)=\left(71^{\circ}, 91^{\circ}\right)$, which is extremely close to the north ecliptic pole at $(\theta, \varphi)=\left(60^{\circ}, 96^{\circ}\right)$. But even more, the two clusters seem to lie on a great circle, hence defining a preferred plane in the sky. The direction perpendicular to this preferred plane has a northern end position at $(\theta, \varphi)=\left(34^{\circ}, 331^{\circ}\right)$ which is very close to the CMB dipole axis at $(\theta, \varphi)=\left(42^{\circ}, 264^{\circ}\right)$ and to the so-called axis of evil. Let us emphasise that also at scale $a_{4}$ (around $10^{\circ}$ of angular size) the CMB presents the same anomaly pattern. In this case there are 11 anomalous directions with a total weight $D_{a_{4}}(\omega)$ larger than the one obtained with any of the 10000 simulations.

In a forthcoming paper (39), we investigate possible origins for this anomaly. For instance, one interesting question is to investigate whether this anomalous detection is due to some structures spatially localised or, on the contrary, the origin for such anomaly is homogeneously distributed in the sky. To answer that question, we have plotted in Figure 6 the pixels that are aligned towards any of the 20 anomalous direction. One can infer that the latter hypothesis is the most plausible one.

We are also analysing whether the origin of the anomaly could be caused by a residual foreground contribution on the CMB map. If so, one should expect to find a frequency dependence for the anomaly, as the foregrounds themselves are frequency dependent. In Figure 7 we plot the $D_{a_{3}}(\omega)$ signal, in $\sigma_{a_{3}}$ units, obtained for each of the Q, V and W WMAP frequency channels. The pattern already shown in Figure 5 is as well observed on each one of the analysed frequencies, indicating that the anomaly seems to be not caused by

$\overline{1}$ The mean direction is obtained by weighting each direction by its corresponding total weight. 

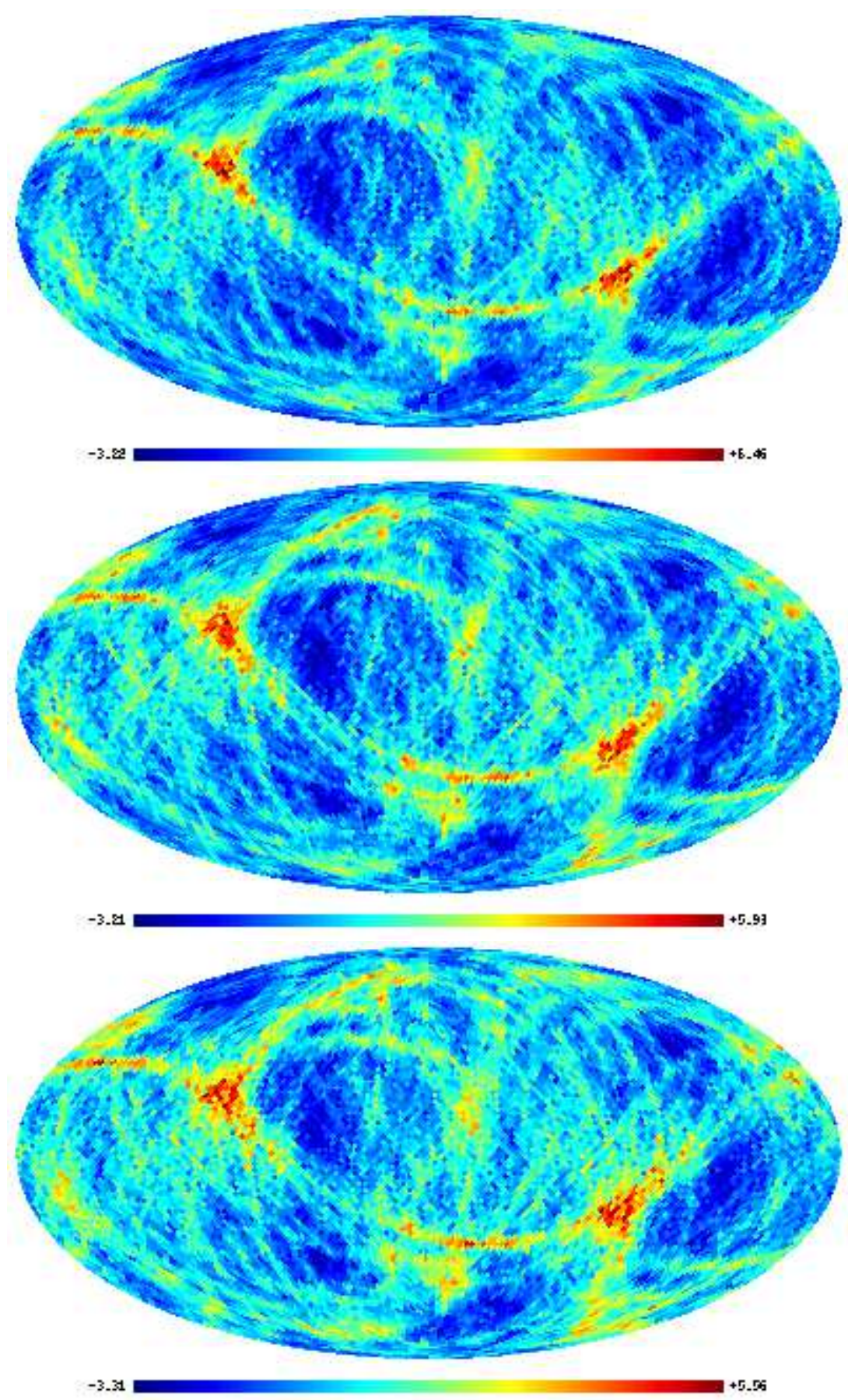

Fig. 7. The total weight $D_{a_{3}}(\omega)$ are plotted in $\sigma_{a_{3}}$ units for the Q (top), V (middle) and W (bottom) WMAP frequency maps. The obtained pattern is the same for all of them and is the same as the one already shown in Figure 5. This fact seems to discard the foregrounds as responsible for the anomaly.

foregrounds.

Finally, the preliminary analysis of the WMAP 3-year data also seems to indicate the same structure of anomalies at the same scales. For comparison 


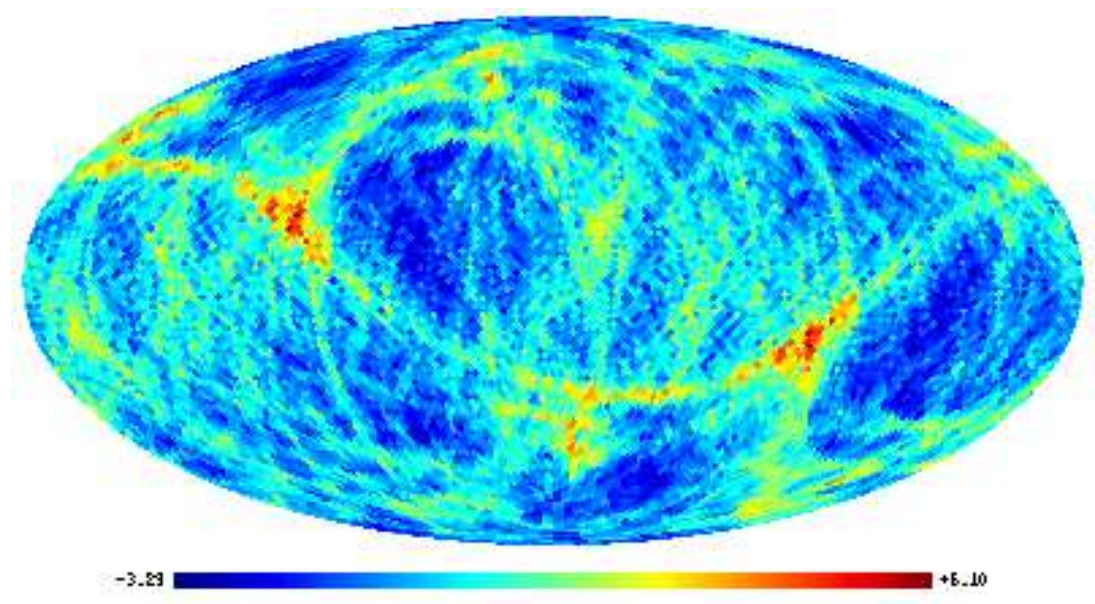

Fig. 8. Map of total weights $D_{a_{3}}(\omega)$ resulting from the WMAP 3-year data at scale $a_{3}$ in $\sigma_{a_{3}}$ units. The same pattern as in the first-year data analysis is observed, although the significance of the anomaly has slightly decreased.

with the results for the first-year data (Figure 4, bottom panel), in Figure 8 we present the $D_{a_{3}}(\omega)$ in $\sigma_{a_{3}}$ units for the 3-year data. The same pattern can be recognised in both data sets, although for the 3-year data the significance of the anomaly seems to be slightly smaller than in the first-year data: whereas in the first release 27 positions were anomalous at $99.99 \%$ (i.e., at maximum, only one simulation has a larger value), in the second one, only 15 positions had a total weight with the same significant. The number of simulations having at least 15 positions at that significance or above is 42 .

\section{Conclusions}

This contribution reviews a novel methodology for probing the universe isotropy through the statistical isotropy of the CMB. It relies on the analysis of the alignment of local features in the CMB proposed by the authors in (38). The power of the method is based on the use of steerable wavelets. On the one hand, steerability makes feasible the computation of the local orientation of the CMB features which is otherwise unaffordable in terms of computation times. On the other hand, the steerability renders accessible the computation of the local orientation of the CMB features, which is otherwise unaffordable in terms of computation times. On the other hand, the scale-space nature of the wavelet scheme allows to explore the alignment of the local structures at different scales, probing possible different phenomena.

In the first application of the method (38) to the WMAP first-year data, we found an extremely significant anisotropy. 20 anomalous directions were identified in the sky at a very high confidence level $(\geq 99.99 \%)$ at a scale $a_{3}$ corresponding to an angular size of $8.3^{\circ}$, corresponding to a multipole range 
between $\ell=11$ and $\ell=27$. The 20 anomalous directions where located in two clusters of 4 and 16 directions respectively. The mean position of the biggest one is at $(\theta, \varphi)=\left(71^{\circ}, 91^{\circ}\right)$, very close to the north ecliptic pole $(\theta, \varphi)=\left(60^{\circ}, 96^{\circ}\right)$. In addition, the two clusters are aligned in the same plane, with a northern end normal direction pointing towards $(\theta, \varphi)=\left(34^{\circ}, 331^{\circ}\right)$, very close to the $\mathrm{CMB}$ dipole axis $(\theta, \varphi)=\left(42^{\circ}, 264^{\circ}\right)$ and the so-called axis of evil. This result synthesised for the first time previously reported anomalies identified towards the dipole and the ecliptic poles axes.

We are currently analysing the origin of such an anomaly (39) as well as its significance in the WMAP 3-year data. The second WMAP data release provides a pattern of total weights compatible with the one obtained with the first one. However, the significance of the detection seems to be slightly lower. We are investigating whether this anomalous detection is due to some structures spatially localised or, on the contrary, the origin for such anomaly is homogeneously distributed in the sky. Our analysis indicates that the latter is the most plausible option. We are also analysing if the origin of the anomaly could be caused by a residual foreground contribution on the CMB map. If so, one should expect to find a frequency dependence for the anomaly, as the foregrounds themselves are frequency dependent. The same pattern of total weights has been found in the individual $\mathrm{Q}, \mathrm{V}$ and $\mathrm{W}$ frequency channels of the first-year data. This might discard foregrounds as a possible origin of such anomaly.

Other possible sources for explaining the anomaly are being considered. Firstly, the coincidence of the preferred direction (towards the ecliptic poles and the CMB dipole axes) naturally suggest possible unknown systematic effects (48; 49). Among them, errors in the beam pattern reconstruction are being studied. It is also interesting to note that the angular size of the mesh of the WMAP scan pattern defined by the combination of the spin and precession of the satellite is of the order of several degrees (50). Second, the angular size at which the anomaly is detected is compatible with the size of primary CMB anisotropies due to topological defects such as texture fields (51) or secondary anisotropies due to the Rees-Sciama effect (52). Alignment mechanisms (48; 53) were recently proposed which might be generalised to such structures.

The authors acknowledge the use of the LAMBDA archive, and of the HEALPix and CMBFAST softwares. YW was supported by the Swiss and Belgian National Science Foundations. PV and EMG were supported by the Spanish MEC project ESP2004-07067-C03-01. 


\section{References}

[1] C. L. Bennet et al., Astrophys. J. Suppl. 148, 1 (2003).

[2] D. N. Spergel et al., Astrophys. J. Suppl. 148, 175 (2003).

[3] D. N. Spergel et al., submitted, preprint LAMBDA web site (2006).

[4] M. Tegmark et al., Phys. Rev. D 69, 103501 (2004).

[5] H. K. Eriksen, F. K. Hansen, A. J. Banday, K. M. Górski, and P. B. Lilje, Astrophys. J. 605, 14 (2004).

[6] H. K. Eriksen, A. J. Banday, K. M. Górski, and P. B. Lilje, Astrophys. J. 622, 58 (2005).

[7] F. K. Hansen, P. Cabella, D. Marinucci, and N. Vittorio, Astrophys. J. Lett. 607, L67 (2004).

[8] F. K. Hansen, K. M. Górski, and E. Hivon, Mon. Not. R. Astron. Soc. 336, 1304 (2002).

[9] F. K. Hansen, A. J. Banday, and K. M. Górski, Mon. Not. R. Astron. Soc. 354, 641 (2004).

[10] F. K. Hansen, A. Balbi, A. J. Banday, and K. M. Górski, Mon. Not. R. Astron. 354, 905 (2004).

[11] E. P. Donoghue and J. F. Donoghue, Phys. Rev. D 71, 043002 (2005).

[12] K. Land and J. Magueijo, Mon. Not. R. Astron. Soc. 357, 994 (2005).

[13] C. J. Copi, D. Huterer, and G. D. Starkman, Phys. Rev. D 70, 043515 (2004).

[14] G. Katz and J. Weeks, Phys. Rev. D 70, 063527 (2004).

[15] D. J. Schwarz, G. D. Starkman, D. Huterer, and C. J. Copi, Phys. Rev. Lett. 93, 221301 (2004).

[16] K. Land and J. Magueijo, Mon. Not. R. Astron. Soc. 362, L16 (2005).

[17] K. Land and J. Magueijo, Mon. Not. R. Astron. Soc. 362, 838 (2005).

[18] A. de Oliveira-Costa, M. Tegmark, M. Zaldarriaga, and A. Hamilton, Phys. Rev. D 69, 063516 (2004).

[19] K. Land and J. Magueijo, Phys. Rev. Lett. 95, 071301 (2005).

[20] P. Bielewicz, H. K. Eriksen, A. J. Banday, K. M. Górski, and P. B. Lilje, Astrophys. J. 635, 750 (2005).

[21] P. Vielva, E. Martínez-González, R. B. Barreiro, J. L. Sanz, and L. Cayón, Astrophys. J. 609, 22 (2004).

[22] M. Cruz, E. Martínez-González, P. Vielva, and L. Cayón, Mon. Not. R. Astron. Soc. 356, 29 (2005).

[23] L. Cayón, J. Jin and A. Treaster, Mon. Not. R. Astron. Soc. 362, 826 (2005).

[24] M. Cruz, Tucci M., E. Martínez-González, P. Vielva, Mon. Not. R. Astron. Soc. 369, 57 (2006).

[25] M. Cruz, L. Cayón, E. Martínez-González, P. Vielva, and J. Jin, Astrophys. J. submitted, preprint astro-ph/0603367 (2006).

[26] E. Martínez-González, M. Cruz, L. Cayón, P. Vielva, and J. Jin, New Astron. Rev. in this volumne, (2006).

[27] A. Bernui, B. Mota, M. J. Rebouas, and R. Tavakol, preprint 
astro-ph/0511666 (2005).

[28] A. Hajian and T. Souradeep, Astrophys. J. Lett. 597, L5 (2003).

[29] A. Hajian, T. Souradeep, and N. Cornish, Astrophys. J. Lett. 618, L63 (2005).

[30] A. Hajian and T. Souradeep, preprint astro-ph/0501001 (2005).

[31] A. Hajian and T. Souradeep, preprint astro-ph/0607153 (2006).

[32] T. R. Jaffe, A. J. Banday, H. K. Eriksen, K. M. Górski, and F. K. Hansen, Astrophys. J. Lett. 629, L1 (2005).

[33] T. R. Jaffe, A. J. Banday, H. K. Eriksen, K. M. Górski, and F. K. Hansen, Astrophys. J. 643, 616 (2006).

[34] T. R. Jaffe, S. Hervik, A. J. Banday and K. M. Górski, Astrophys. J. 644, 701 (2006).

[35] L. Cayón, A. J. Banday, T. R. Jaffe, H. K. Eriksen, F. K. Hansen, K. M. Górski and J. Jin, Mon. Not. R. Astron. Soc. 369, 598 (2006).

[36] J. D. McEwen, M. P. Hobson, A. N. Lasenby and D. J. Mortlock, Mon. Not. R. Astron. Soc. 369, 1858 (2006).

[37] M. Bridles, J. D. McEwen, A. N. Lasenby and M. P. Hobson, Mon. Not. R. Astron. Soc. submitted, preprint astro-ph/0605325, (2006).

[38] Y. Wiaux, P. Vielva, E. Martínez-González and P. Vandergheynst, Phys. Rev. Lett., 96, 151303 (2006).

[39] P. Vielva, Y. Wiaux, E. Martínez-González and P. Vandergheynst, to be submitted (2006).

[40] Y. Wiaux, L. Jacques, P. Vielva and P. Vandergheynst, Astrophys. J. accepted, preprint astro-ph/0508516 (2006).

[41] Y. Wiaux, L. Jacques, and P. Vandergheynst, J. Comput. Phys. submitted, preprint astro-ph/0508514 (2006)

[42] W. T. Freeman and E. H. Adelson, IEEE Trans. Pattern Anal. Machine Intell., 13, 891, (1991).

[43] Y. Wiaux, L. Jacques, and P. Vandergheynst, Astrophys. J. 632, 15 (2005).

[44] E. Komatsu et al., Astrophys. J. Suppl. 148, 119 (2003).

[45] C. L. Bennet et al., Astrophys. J. Suppl. 148, 97 (2003).

[46] K. M. Górski, E. Hivon, A. J. Banday, B. D. Wandelt, F. K. Hansen, M. Reinecke, and M. Bartelman, Astrophys. J. 622, 759 (2005).

[47] U. Seljak \& M. Zaldarriaga, Astrophys. J., 469, 437 (1996)

[48] C. Gordon, W. Hu, D. Huterer, and T. Crawford, Phys. Rev. D 72, 103002 (2005).

[49] P. E. Freeman, C. R. Genovese, C. J. Miller, R. C. Nichol, and L. Wasserman, Astrophys. J. 638, 1 (2006).

[50] C. L. Bennet et al., Astrophys. J. 583, 1 (2003).

[51] N. Turok and D. N. Spergel, Phys. Rev. Lett. 64, 2736 (1990).

[52] E. Martínez-González and J. L. Sanz, Mon. Not. R. Astron. Soc. 247, 473 (1990).

[53] A. Rakić, S. Räsänen, and D. J. Schwarz, Mon. Not. R. Astron. Soc. 369, 27 (2006). 